\title{
Emergence of BCR-ABL Kinase Domain Mutations Associated with Newly Diagnosed Chronic Myeloid Leukemia: A Meta-Analysis of Clinical Trials of Tyrosine Kinase Inhibitors
}

\author{
Iulia D. Ursan, PharmD; Ruixuan Jiang, BS; Evan M. Pickard; Todd A. Lee, PharmD, PhD; \\ Daniel Ng, PharmD, MBA; and A. Simon Pickard, PhD
}

\begin{abstract}
BACKGROUND: Tyrosine kinase inhibitors (TKIs) are a mainstay of treatment for patients suffering from chronic myeloid leukemia (CML). Testing for various mutations in the BCR-ABL gene may help predict lack of response to specific TKIs where resistance has developed.

OBJECTIVE: To estimate the emergence of BCR-ABL kinase domain mutations associated with newly diagnosed $C M L$ patients exposed to first-line TKI treatment.

METHODS: Published studies were identified using a structured search of online databases. Original research studies were included if they reported the incidence of specific BCR-ABL kinase domain point mutations after first-line TKI treatment failure or baseline mutations for second-line TKI treatment following first-line treatment failure. Meta-analysis of mutation rates across studies was based on DerSimonian and Laird's randomeffects model.
\end{abstract}

RESULTS: Of 1,323 citations, 12 studies met the inclusion criteria, involving a total of 1,698 patients. Overall mutation rates $(95 \% \mathrm{Cl})$ were imatinib 9.7\% (6.2\%-13.3\%); dasatanib 1.7\% (0.0\%-4.3\%); and nilotinib $3.3 \%$ (0.0\%-7.7\%). The most common specific mutations were T315I, E255K, and M351T. T315I mutations constituted $58 \%$ (7 of 12) of dasatinib-related mutations and 13\% (15 of 117) of imatinib-related mutations.

CONCLUSIONS: Lack of response to TKIs associated with mutation in the BCR-ABL gene was significantly higher in imatinib-treated patients, and all mutations arose after treatment. T315I was a common treatment-emergent mutation. Further research is needed to assess the prognostic value of testing for mutations and the economic implications of treatment-emergent mutations.

J Manag Care Pharm. 2015;21(2):114-22

Copyright $\odot 2015$, Academy of Managed Care Pharmacy. All rights reserved.

\section{What is already known about this subject}

Tyrosine kinase inhibitors (TKIs) have revolutionized the management and outcome of chronic myeloid leukemia (CML), and they are a mainstay of treatment for these patients.

Development of TKI resistance due to mutation is a critical issue for some patients and can result in additional costs.

Testing for various mutations in the BCR-ABL gene may help predict lack of response to specific TKIs where resistance has developed and may inform the choice between alternative TKIs in CML patients.

\section{What this study adds}

This study employed a meta-analytic approach to estimate the incidence of mutations associated with TKIs.

The incidence of mutation in the BCR-ABL gene was significantly higher among patients exposed to imatinib compared with dasatanib and nilotinib.

The most common mutations (>1\%) were T315I, E255K, and M351T.

These findings may assist provider decision making regarding TKI treatment for CML patients and provide inputs for rates of mutation for cost-effectiveness analysis of TKIs.

T yrosine kinase inhibitors (TKIs) act by preventing disease progression through the inhibition of BCR-ABL kinase activity and decreasing the number of malignant cells. ${ }^{1,2}$ As a first-generation TKI, imatinib has demonstrated superior hematologic and cytogenetic responses (HR and CyR, respectively) when compared with interferon- $\alpha$ plus cytarabine. ${ }^{3}$ Although these results indicate that imatinib may significantly prolong event-free survival in chronic myeloid leukemia (CML) patients, they may develop intolerance or resistance to the medication. ${ }^{4-6}$ Approximately one-third of imatinib-treated CML patients do not achieve complete CyR (CCyR), and up to $50 \%$ of the imatinib resistant cases may result from mutations in the BCR-ABL tyrosine kinase (TK), ,,$-9^{-9}$

Imatinib has also been shown to be less effective in patients who have progressed past the initial chronic phase $(\mathrm{CP})$ to more advanced disease stages, such as the accelerated and blast phases of CML (AP and BP, respectively). ${ }^{10}$ In addition to imatinib, several TKIs have recently emerged on the market, including bosutinib, dasatanib, nilotinib, and ponatinib. These medications tend to be used as second- or third-line agents after treatment failure with imatinib due to resistance, disease progression, or intolerance. ${ }^{11}$ These agents can inhibit the BCRABL protein kinase and may also effectively target additional disease progression pathways.

Various mechanisms are thought to mediate resistance to TKI treatment. The most common of these mechanisms is BCR-ABL kinase domain mutations, causing patients to 
respond inadequately to the TKI or relapse during treatment. Testing for various mutations in the BCR-ABL kinase domain may help indicate a lack of response to TKIs when resistance has developed and may inform the choice between alternative TKIs. ${ }^{12}$ Although one of the most widely reported BCR-ABLrelated mutations is the T315I mutation, many types of mutations may be associated with treatment failure..$^{11-14}$ Although clinical studies often report the incidence of various types of mutations among patients who fail therapy or complete a trial, specific types of mutations are relatively rare, and most trials involve no more than several hundred patients. Thus, estimates of the incidence of specific mutations vary widely. We sought to employ a meta-analytic approach to estimate incidence of mutations associated with TKIs across the published literature using criteria intended to minimize sources of heterogeneity in study design, endpoints, and treatment failure definitions.

Our specific purpose was to estimate the incidence of mutations in the BCR-ABL kinase domain among patients exposed to first-line TKI therapy for CML.

\section{Methods}

A systematic literature search was performed in MEDLINE (Medical Literature Analysis and Retrieval System Online), CINAHL (Cumulative Index to Nursing and Allied Health Literature), IPA (International Pharmaceutical Abstracts), and EMBASE (Excerpta Medica dataBASE) using the following terms: chronic myeloid leukemia AND (tyrosine kinase inhibitor OR imatinib OR ponatinib OR nilotinib OR dasatinib OR bosutinib). Criteria for inclusion were restricted to original research published between January 1, 1966, and May 31, 2013, that were clinical trials conducted in humans and written in the English language.

Following initial screening, full papers were retrieved and additional screening criteria applied. To be included, studies had to be or have done the following:

1. Original research investigating TKI use in the treatment of CML.

2. Test for the incidence of genetic mutations after first-line treatment failure, which could also include studies testing for presence of mutation prior to initiation of second-line treatments.

3. Report the number of patients initially exposed to first-line treatment in either the text or in a referenced study.

4. Include 10 or more patients in the study.

5. Use an endpoint that included major cytogenetic response (MCyR) or CCyR; disease progression to subsequent phases of CML (i.e., CP to AP/BP); or another form of TKI treatment failure that could include treatment discontinuation or switch, death, or hematopoietic stem cell transplantation.

Individuals were stratified by BCR-ABL kinase domain point mutations for each definition of TKI treatment failure. Patients who did not achieve MCyR or CCyR through the end of follow-up were defined as having failed treatment. When several articles reported results from the same dataset, only 1 article was included. The unit of analysis was type of specific mutation; therefore, in the case of compound mutations, a single patient could contribute 2 or more mutations to the analysis. However, it is important to point out that not all patients in a given study were evaluated for mutations.

A standardized data abstraction form was developed to extract information from the eligible studies. Two reviewers independently extracted the data from each of the studies. Disagreements were resolved by consensus with input from a third reviewer.

Results were reported for each TKI separately. The incidence of mutations were estimated in 2 ways: (1) concerning specific types of mutation, calculated as a simple proportion of the number of patients with a genetic mutation divided by the total population exposed to the TKI (as presented in Table 3 and Appendices A and B, available in online article) and (2) concerning overall incidence of mutation, using the randomeffects (DerSimonian and Laird) method for meta-analysis. ${ }^{15}$ Rates of genetic mutations were referred to as incidence rates because they appeared to emerge after baseline (prior to treatment), although a confluence of factors potentially contributed to their emergence, including treatment exposure. Significant differences in the incidence of mutations between TKIs were examined using the chi-square test. $P$ values $<0.05$ were considered statistically significant.

The heterogeneity of the studies was assessed using the indicator $\mathrm{I}^{2}$. Possible sources of heterogeneity were assessed in the meta-analysis, and studies were stratified based on different definitions of treatment failure, inclusion of advanced phases, and different follow-up durations. STATA 11 (StataCorp, College Station, TX) was used to conduct the statistical analysis.

\section{Results}

From 1,323 citations identified using the initial search terms, 12 studies met the inclusion criteria, involving a total of 1,698 patients. From the 611 citations, 377 abstracts were excluded based on abstract review. The remaining 234 full-text articles were retrieved and screened. Of these, 225 articles were excluded because they were not an original research article identifying mutations among CML patients first treated with a TKI. Three additional articles were identified through crossreferences, resulting in a total of 12 articles that ultimately met our inclusion criteria (Figure 1). Of these, 10 trials focused on imatinib, of which 2 also involved dasatinib. ${ }^{16,17}$ In total, 3 studies examined dasatinib as front-line therapy, ${ }^{16-18}$ and 1 study involved nilotinib. ${ }^{19}$ The majority of the studies were publicly funded.

The study publication years ranged from 2002-2013 (Table 1). Five studies were multicenter, and 7 studies were single center. All of the studies timed the mutation analysis at treatment failure or discontinuation. The studies varied in their 


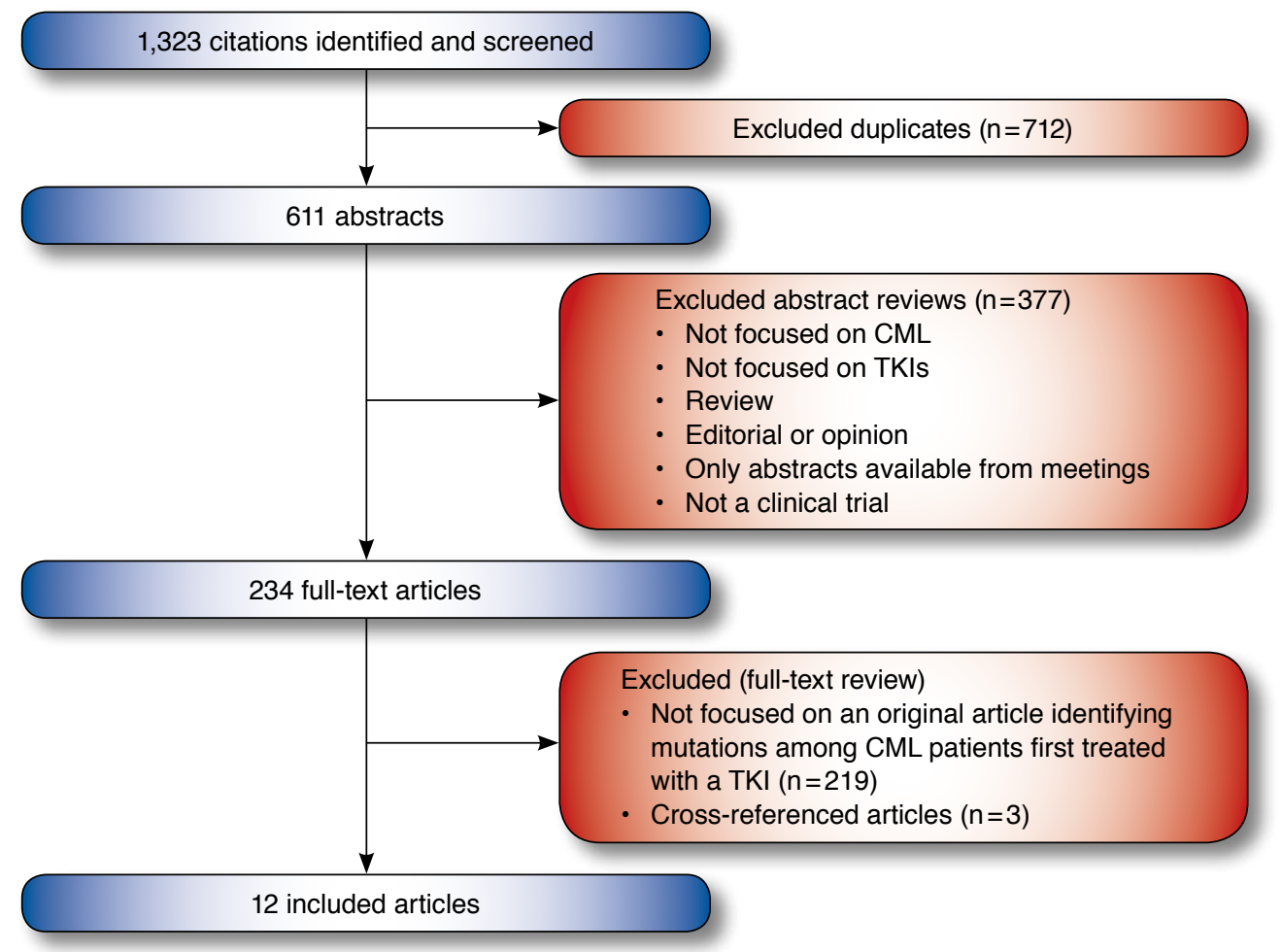

$C M L=$ chronic myeloid leukemia; $T K I=$ tyrosine kinase inhibitor.

definitions of treatment failure, but most defined failure or subobptimal response as the inability to achieve complete hematological remission (CHR), MCyR, or CCyR, or the experience of disease progression after a specified time period of treatment (generally 3,6 , or 12 months).

The included studies involved 1,698 patients who were mutation tested (1,277 imatinib-treated, 360 dasatanib-treated, and 61 nilotinb-treated patients; Table 2). The number of patients in each treatment arm ranged from 9 to 258 people. The overall median age was 47 years. The age range of the patient cohort was 18 to 90 years. The proportion of males ranged from $52.5 \%-63.0 \%$. The median duration of follow-up was 32.5 months. All studies included patients in CP CML, and 4 studies also included patients in AP, with 1 trial including patients in blast crisis. No mutations at baseline were identified.

Ten studies screened imatinib-treated patients for mutations (Table 3). The overall incidence of mutation in patients who received imatinib as first-line therapy was 9.74\% (95\% confidence interval $[\mathrm{CI}]=6.2 \%-13.3 \%$; Figure 2 ). The most commonly identified mutations were $\operatorname{E255K}(\mathrm{n}=16$, incidence $=1.3 \%) ; \quad$ M351T $\quad(n=15, \quad$ incidence $=1.2 \%) ; \quad F 359 V$ $(n=11$, incidence $=0.9 \%)$; and G250E $(n=9$, incidence $=0.6 \%)$. Regarding T315I, across studies there were 15 patients with the treatment-emergent $\mathrm{T} 315 \mathrm{I}$ mutation (incidence $=1.2 \%$ ). Overall, no F317C, F317V, F317I, or T315A mutations were reported. The incidence of mutation ranged from 0\% (T315A) to $1.3 \%$ (E255K). Eight studies reported treatment failure as defined by MCyR or CCyR failure. The most prevalent mutations among those who failed to achieve MCyR were M244V $(\mathrm{n}=2$, incidence $=0.2 \%)$ and E255K ( $\mathrm{n}=3$, incidence $=0.3 \%)$. The most common mutations in patients who failed to achieve CCyR were G250E ( $n=2$, incidence $=0.2 \%)$ and $F 359 \mathrm{~V}(\mathrm{n}=2$, incidence $=0.2 \%$ ). For T315I, 4 patients had treatment-emergent T315I mutation (incidence $=0.4 \%$ ).

M351T, E255K, and F359V mutations occurred most frequently for patients who had a more general definition of treatment failure (not specifically failing MCyR or CCyR), treatment discontinuation, treatment switch, disease progression, or death. The mutation incidence of M351T was 1.1\% $(n=14)$; E255K was $0.9 \%(n=12)$; and $F 359 V$ was $0.7 \%(n=9)$. Treatment-emergent T315I mutation occurred in 13\% of imatinib-treated patients (15 of 115) who experienced a mutation. The heterogeneity indicator $\mathrm{I}^{2}$ was high for the imatinib studies at $80.6 \%$.

Three dasatinib studies included 360 patients (Appendix A, available in online article). ${ }^{16-18}$ Two studies detected V299L in 2 patients - 1 patient failed to reach or maintain 


\begin{tabular}{|c|c|c|c|c|c|c|c|c|}
\hline First Author & Year & Trial Sponsor & $\begin{array}{l}\text { Multi- } \\
\text { center }\end{array}$ & $\begin{array}{l}\text { Method of } \\
\text { Mutational } \\
\text { Analysis }\end{array}$ & $\begin{array}{l}\text { Timing of } \\
\text { Mutation } \\
\text { Analysis }\end{array}$ & Study Definition of Failure & Medication & Dosing \\
\hline \multirow[t]{2}{*}{ Radich, J17 } & \multirow[t]{2}{*}{2012} & \multirow{2}{*}{$\begin{array}{c}\text { Canadian } \\
\text { Cancer Society } \\
\text { Research } \\
\text { Institute, } \\
\text { NIH, Public } \\
\text { Health Service } \\
\text { Cooperative } \\
\text { Agreement }\end{array}$} & \multirow[t]{2}{*}{ Yes } & \multirow[t]{2}{*}{$\begin{array}{c}\text { Direct } \\
\text { nucleotide } \\
\text { sequencing }\end{array}$} & $\begin{array}{l}\text { Tx failure or } \\
\text { discontinuation }\end{array}$ & \multirow[t]{2}{*}{$\begin{array}{l}\text { Failure to achieve CHR by } 3 \text { months, } \\
\text { loss of CHR, loss of PCyR or CCyR, or } \\
\text { progression to AP or BP CML }\end{array}$} & Imatinib & $400 \mathrm{mg} \mathrm{D}$ \\
\hline & & & & & $\begin{array}{l}\text { Tx failure or } \\
\text { discontinuation }\end{array}$ & & Dasatinib & $100 \mathrm{mg} D$ \\
\hline Soverini, S38 & 2005 & $\begin{array}{l}\text { European } \\
\text { Leukemia Net } \\
\text { and others }\end{array}$ & Yes & $\begin{array}{l}\text { D-HPLC } \\
\text { or direct } \\
\text { sequencing }\end{array}$ & $\begin{array}{c}\text { Baseline/ } \\
\text { scheduled/ } \\
\text { tx failure/ } \\
\text { discontinuation }\end{array}$ & $\begin{array}{l}\text { Cytogenetic resistance: failure to obtain } \\
\text { at least a MCyR at } 12 \text { months }\end{array}$ & Imatinib & $400 \mathrm{mg} \mathrm{D}$ \\
\hline Branford, S39 & 2007 & $\begin{array}{l}\text { Novartis } \\
\text { Australia }\end{array}$ & Yes & $\begin{array}{c}\text { Direct } \\
\text { sequencing }\end{array}$ & $\begin{array}{c}\text { Scheduled/ } \\
\text { tx failure/ } \\
\text { discontinuation }\end{array}$ & $\begin{array}{l}\text { Death from any cause, development of } \\
\text { AP or blast crisis, loss of CHR, and loss } \\
\text { of a MCyR }\end{array}$ & Imatinib & $400 \mathrm{mg} \mathrm{D}$ \\
\hline Branford, $\mathrm{S}^{30}$ & 2003 & $\begin{array}{l}\text { Novartis } \\
\text { Australia }\end{array}$ & Yes & $\begin{array}{l}\text { Direct } \\
\text { sequencing }\end{array}$ & $\begin{array}{l}\text { Scheduled/ } \\
\text { tx failure/ } \\
\text { discontinuation }\end{array}$ & $\begin{array}{l}\text { CP: disease progression, loss of a sus- } \\
\text { tained CHR, MCyR, or CCyR with a cor- } \\
\text { responding increase in BCR-ABL levels } \\
\text { of at least } 1 \text { log (refer to the reference for } \\
\text { the definitions of acquired and primary } \\
\text { resistance in CP or AP) }\end{array}$ & Imatinib & $400 \mathrm{mg} \mathrm{D}$ \\
\hline de Lavallade, $\mathrm{H}^{40}$ & 2008 & NR & No & $\begin{array}{c}\text { RQ-PCR and } \\
\text { pyrosequencing }\end{array}$ & $\begin{array}{l}\text { Scheduled/ } \\
\text { tx failure/ } \\
\text { discontinuation }\end{array}$ & $\begin{array}{l}\text { Loss of CHR or progression to advanced } \\
\text { phase, loss of MCyR or CCyR, an } \\
\text { increase by a factor of } 2 \text { in the transcript } \\
\text { level, and failure to achieve CHR by } 12 \\
\text { weeks }\end{array}$ & Imatinib & $400 \mathrm{mg} \mathrm{D}$ \\
\hline $\mathrm{He}, \mathrm{H}^{20}$ & 2012 & NR & No & $\begin{array}{l}\text { Semi-nested } \\
\text { transcriptase } \\
\text { PCR followed } \\
\text { by direct } \\
\text { sequencing }\end{array}$ & $\begin{array}{c}\text { Baseline/ } \\
\text { scheduled/ } \\
\text { tx failure/ } \\
\text { discontinuation }\end{array}$ & $\begin{array}{l}\text { Loss of CCyR or MCyR, MCyR not } \\
\text { achieved within } 12 \text { months, and pro- } \\
\text { gression to advanced phase }\end{array}$ & Imatinib & $400 \mathrm{mg} \mathrm{D}$ \\
\hline \multirow[t]{2}{*}{ Kantarjian, $\mathrm{H}^{16}$} & \multirow[t]{2}{*}{2012} & \multirow[t]{2}{*}{$\begin{array}{l}\text { Bristol-Myers } \\
\text { Squibb }\end{array}$} & \multirow[t]{2}{*}{ Yes } & \multirow[t]{2}{*}{$\begin{array}{c}\text { Direct } \\
\text { sequencing }\end{array}$} & $\begin{array}{c}\text { Tx failure/ } \\
\text { discontinuation }\end{array}$ & \multirow{2}{*}{$\begin{array}{l}\text { No hematologic response by } 3 \text { months, } \\
\text { no CHR or cytogenetic response by } 6 \\
\text { months, no partial cytogenetic response } \\
\text { by } 12 \text { months, or no CCyR by } 18 \\
\text { months }\end{array}$} & Imatinib & $400 \mathrm{mg} \mathrm{D}$ \\
\hline & & & & & $\begin{array}{l}\text { Tx failure/ } \\
\text { discontinuation }\end{array}$ & & Dasatinib & $100 \mathrm{mg} D$ \\
\hline Roche-Lestienne, $C^{13}$ & 2002 & NR & No & Sequencing & $\begin{array}{c}\text { Scheduled/ } \\
\text { tx failure/ } \\
\text { discontinuation }\end{array}$ & No cytogenetic response after 3 months & Imatinib & $\begin{array}{l}400-800 \\
\text { mg D }\end{array}$ \\
\hline Elnahass, $\mathrm{Y}^{33}$ & 2013 & NR & No & Sequencing & $\begin{array}{c}\text { Scheduled/ } \\
\text { tx failure/ } \\
\text { discontinuation }\end{array}$ & $\begin{array}{l}\text { Greater than 2-fold rising BCR-ABL } \\
\text { level }\end{array}$ & Imatinib & $400 \mathrm{mg} \mathrm{D}$ \\
\hline Wei, $\mathrm{Y}^{41}$ & 2006 & $\begin{array}{l}\text { Swedish } \\
\text { Research } \\
\text { Council and } \\
\text { others }\end{array}$ & No & Sequencing & $\begin{array}{c}\text { Baseline/ } \\
\text { scheduled/ } \\
\text { tx failure/ } \\
\text { discontinuation }\end{array}$ & $\begin{array}{l}\text { Failure to achieve CHR after } 3 \text { months } \\
\text { of imatinib therapy, failure to achieve } \\
\text { at least a minimal cytogenetic response } \\
\text { ( } 65 \%-95 \% \text { Ph+ metaphases) after } 6 \\
\text { months of imatinib therapy, failure to } \\
\text { achieve a MCyR }<35 \% \text { Ph+ metaphases } \\
\text { at } 12 \text { months of imatinib therapy } \\
\text { (1-3=primary resistance), and loss of an } \\
\text { earlier obtained CHR or CyR (acquired } \\
\text { resistance) }\end{array}$ & Imatinib & $\begin{array}{l}400-600 \\
\text { mg D }\end{array}$ \\
\hline \multirow[t]{2}{*}{ Cortes, J19 } & \multirow[t]{2}{*}{2010} & \multirow[t]{2}{*}{$\begin{array}{l}\text { National } \\
\text { Cancer } \\
\text { Institute }\end{array}$} & \multirow[t]{2}{*}{ No } & \multirow[t]{2}{*}{ Sequencing } & \multirow[t]{2}{*}{$\begin{array}{c}\text { Baseline/ } \\
\text { scheduled/ } \\
\text { tx failure/ } \\
\text { discontinuation }\end{array}$} & $\begin{array}{l}\text { Patients who had not achieved CCyR by } \\
6 \text { months after treatment or who devel- } \\
\text { oped resistance }\end{array}$ & \multirow[t]{2}{*}{ Nilotinib } & \multirow[t]{2}{*}{$\begin{array}{l}400 \mathrm{mg} \\
\text { BID }\end{array}$} \\
\hline & & & & & & $\begin{array}{l}\text { Patients who do not achieve MCyR } \\
\text { or patients who discontinue therapy } \\
\text { because of toxicity are also considered } \\
\text { as experiencing treatment failure }\end{array}$ & & \\
\hline Cortes, J18 & 2010 & $\begin{array}{l}\text { National } \\
\text { Cancer } \\
\text { Institute }\end{array}$ & No & Sequencing & $\begin{array}{c}\text { Baseline/ } \\
\text { scheduled/ } \\
\text { tx failure/ } \\
\text { discontinuation } \\
\end{array}$ & $\begin{array}{l}\text { Patients who had not achieved CCyR by } \\
6 \text { months after treatment or who devel- } \\
\text { oped resistance }\end{array}$ & Dasatinib & $\begin{array}{l}50 \mathrm{mg} \\
\text { BID } \\
\text { or } 100 \\
\mathrm{mg} \mathrm{D} \\
\end{array}$ \\
\hline
\end{tabular}


Emergence of BCR-ABL Kinase Domain Mutations Associated with Newly Diagnosed

Chronic Myeloid Leukemia: A Meta-Analysis of Clinical Trials of Tyrosine Kinase Inhibitors

TABLE 2 Characteristics of Patients in the Included Studies

\begin{tabular}{|c|c|c|c|c|c|c|c|c|}
\hline First Author & Medication & $\begin{array}{c}\text { Total Number } \\
\text { of Patients in } \\
\text { Treatment Arm }\end{array}$ & $\begin{array}{c}\text { Median } \\
\text { Age } \\
\text { (Years) }\end{array}$ & $\begin{array}{c}\text { Age } \\
\text { Range } \\
\text { (Years) }\end{array}$ & $\begin{array}{c}\text { Gender } \\
(\% \text { Male })\end{array}$ & $\begin{array}{c}\text { Median Duration } \\
\text { of Follow-up } \\
\text { (Months) }\end{array}$ & $\begin{array}{l}\text { Median Duration } \\
\text { Until Mutation } \\
\text { Detected (Months) }\end{array}$ & $\begin{array}{l}\text { CML Phase } \\
\text { (at Time of } \\
\text { Study Entry) }\end{array}$ \\
\hline \multirow[t]{2}{*}{ Radich, J17 } & Imatinib & 13 & 50 & $19-89$ & 59 & 36 & NR & \multirow[t]{2}{*}{$\mathrm{CP}$} \\
\hline & Dasatinib & 9 & 47 & $18-90$ & 60 & 36 & NR & \\
\hline Soverini, S38 & Imatinib & 175 & 53 & $29-68$ & 55.6 & 32.5 & 3 & $\mathrm{CP}$ \\
\hline Branford, $\mathrm{S}^{39}$ & Imatinib & 53 & NR & NR & NR & 33 & NR & $\mathrm{CP}$ \\
\hline Branford, $\mathrm{S}^{30}$ & Imatinib & 144 & NR & NR & NR & NR & 8 & $\begin{array}{c}\text { AP (40), late-CP (64), } \\
\text { early-CP (44) }\end{array}$ \\
\hline de Lavallade, $\mathrm{H}^{40}$ & Imatinib & 192 & 46.3 & $18-79$ & 56.9 & 38 & 10.5 & $\mathrm{CP}$ \\
\hline $\mathrm{He}, \mathrm{H}^{20}$ & Imatinib & $210,24,41$ & $\mathrm{NR}$ & $16-80$ & 56.7 & $48,40,17$ & 14.6 & $\mathrm{CP}(76), \mathrm{AP}(9), \mathrm{BC}(15)$ \\
\hline \multirow[t]{2}{*}{ Kantarjian, $\mathrm{H}^{16}$} & Imatinib & 258 & 49 & $18-78$ & 63 & 26.7 & NR & $\mathrm{CP}$ \\
\hline & Dasatinib & 258 & 46 & $18-84$ & 56 & 26.5 & NR & $\mathrm{CP}$ \\
\hline Roche-Lestienne, $\mathrm{C}^{13}$ & Imatinib & 71 & NR & NR & NR & NR & 3 & CP (67), AP (33) \\
\hline Elnahass, $Y^{33}$ & Imatinib & 72 & 42 & $18-72$ & 61.1 & 41.6 & NR & $\mathrm{CP}$ \\
\hline Wei, Y ${ }^{41}$ & Imatinib & 40 & NR & $23-80$ & 52.5 & 24 & 10.5 & CP (92), AP (8) \\
\hline Cortes, J19 & Nilotinib & 61 & 46 & $19-86$ & NR & 17.3 & NR & early CP \\
\hline Cortes, $\mathrm{J}^{18}$ & Dasatinib & 62 & 47 & $18-76$ & NR & 24 & NR & $\mathrm{CP}$ \\
\hline
\end{tabular}

TABLE 3 Mutation Incidence for Imatinib

\begin{tabular}{|c|c|c|c|c|c|c|c|c|c|c|c|c|}
\hline \multirow[b]{3}{*}{$\stackrel{\Xi}{\stackrel{\Xi}{\Xi ี \Xi}}$} & \multicolumn{12}{|c|}{ Criteria Defining Treatment Failure } \\
\hline & \multicolumn{3}{|c|}{ Composite } & \multicolumn{3}{|c|}{ MCyR } & \multicolumn{3}{|c|}{ CCyR } & \multicolumn{3}{|c|}{ Other } \\
\hline & 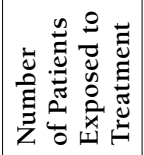 & 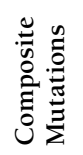 & 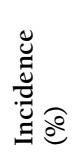 & 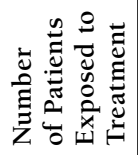 & 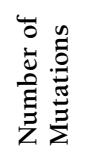 & 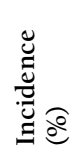 & 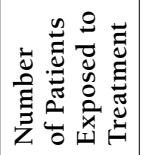 & 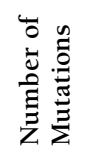 & 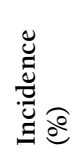 & 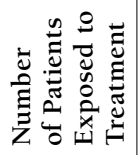 & 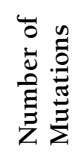 & 苞 \\
\hline $\mathrm{M} 244 \mathrm{~V}$ & 1,277 & 7 & 0.55 & 947 & 2 & 0.21 & 947 & 0 & 0 & 1,277 & 5 & 0.39 \\
\hline L248V & 1,277 & 1 & 0.08 & 947 & 0 & 0 & 947 & 0 & 0 & 1,277 & 1 & 0.08 \\
\hline G250E & 1,277 & 9 & 0.63 & 947 & 1 & 0.11 & 947 & 2 & 0.21 & 1,277 & 6 & 0.47 \\
\hline Q252H & 1,277 & 7 & 0.55 & 947 & 0 & 0 & 947 & 0 & 0 & 1,277 & 7 & 0.55 \\
\hline Y253F & 1,277 & 6 & 0.47 & 947 & 1 & 0.11 & 947 & 0 & 0 & 1,277 & 5 & 0.39 \\
\hline $\mathrm{Y} 253 \mathrm{H}$ & 1,277 & 6 & 0.47 & 947 & 1 & 0.11 & 947 & 0 & 0 & 1,277 & 5 & 0.39 \\
\hline E255K & 1,277 & 16 & 1.25 & 947 & 3 & 0.32 & 947 & 1 & 0.11 & 1,277 & 12 & 0.94 \\
\hline E255V & 1,277 & 2 & 0.16 & 947 & 1 & 0.11 & 947 & 0 & 0 & 1,277 & 1 & 0.08 \\
\hline $\mathrm{D} 276 \mathrm{G}$ & 1,205 & 2 & 0.17 & 947 & 1 & 0.11 & 947 & 0 & 0 & 1,205 & 1 & 0.08 \\
\hline V299L & 1,277 & 1 & 0.08 & 947 & 0 & 0 & 947 & 0 & 0 & 1,277 & 1 & 0.08 \\
\hline T315A & 1,205 & 0 & 0 & 947 & 0 & 0 & 947 & 0 & 0 & 1,205 & 0 & 0 \\
\hline T315I & 1,277 & 15 & 1.17 & 947 & 6 & 0.63 & 947 & 4 & 0.42 & 1,277 & 5 & 0.39 \\
\hline F317L & 1,277 & 4 & 0.31 & 947 & 0 & 0 & 947 & 0 & 0 & 1,277 & 4 & 0.31 \\
\hline F317C & 1,205 & 0 & 0 & 947 & 0 & 0 & 947 & 0 & 0 & 1,205 & 0 & 0 \\
\hline F317I & 1,205 & 0 & 0 & 947 & 0 & 0 & 947 & 0 & 0 & 1,205 & 0 & 0 \\
\hline F317V & 1,205 & 0 & 0 & 947 & 0 & 0 & 947 & 0 & 0 & 1,205 & 0 & 0 \\
\hline M351T & 1,277 & 15 & 1.17 & 947 & 0 & 0 & 947 & 1 & 0.11 & 1,277 & 14 & 1.10 \\
\hline E355G & 1,277 & 7 & 0.55 & 947 & 1 & 0.11 & 947 & 0 & 0 & 1,277 & 6 & 0.47 \\
\hline F359C & 1,205 & 1 & 0.08 & 947 & 0 & 0 & 947 & 0 & 0 & 1,205 & 1 & 0.08 \\
\hline F359V & 1,277 & 11 & 0.86 & 947 & 0 & 0 & 947 & 2 & 0.21 & 1,277 & 9 & 0.70 \\
\hline F359I & 1,205 & 2 & 0.17 & 947 & 0 & 0 & 947 & 0 & 0 & 1,205 & 2 & 0.17 \\
\hline H396R & 1,134 & 3 & 0.26 & 876 & 0 & 0 & 876 & 0 & 0 & 1,134 & 3 & 0.26 \\
\hline E450G & 1,134 & 5 & 0.44 & 876 & 1 & 0.11 & 876 & 1 & 0.11 & 1,134 & 3 & 0.26 \\
\hline F459K & 1,134 & 6 & 0.53 & 876 & 0 & 0 & 876 & 1 & 0.11 & 1,134 & 5 & 0.44 \\
\hline
\end{tabular}


Study

Dasatinib

Kantarjian, H (2012) $)^{16}$

Radich, J (2012) ${ }^{17}$

Cortes, J (2010) ${ }^{18}$

Subtotal $\left(I^{2}=82.4 \%, P=0.003\right)$

\section{Imatinib}

Kantarjian, H (2012) ${ }^{16}$

Wei, $Y(2006)^{41}$

Elnahass, $Y(2013)^{33}$

Roche-Lestienne, C (2002) ${ }^{13}$

$\mathrm{He}, \mathrm{H}(2012)^{20}$

de Lavallade, $\mathrm{H}(2008)^{40}$

Branford, S (2003) $)^{30}$

Branford, S (2007) $)^{39}$

Soverini, S (2005) ${ }^{38}$

Radich, J (2012) $)^{17}$

Subtotal $\left(I^{2}=80.6 \%, P=0.000\right)$

Nilotinib
Cortes, J (2010)
Subtotal
Incidence $(95 \% \mathrm{Cl})$

\begin{tabular}{|c|c|c|}
\hline 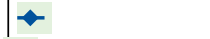 & 0.04 & $(0.02-0.06)$ \\
\hline 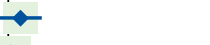 & 0.02 & $(-0.02-0.05)$ \\
\hline 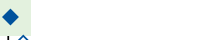 & 0.00 & $(-0.00-0.00)$ \\
\hline 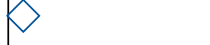 & 0.02 & $(-0.01-0.04)$ \\
\hline$\rightarrow$ & 0.03 & $(0.01-0.06)$ \\
\hline 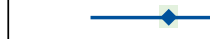 & 0.28 & $(0.14-041)$ \\
\hline$\longrightarrow$ & 0.22 & $(0.13-0.32)$ \\
\hline$\rightarrow$ & 0.07 & $(0.01-0.13)$ \\
\hline$\leftrightarrow$ & 0.08 & (0.05-0.12) \\
\hline 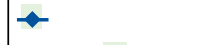 & 0.04 & $(0.01-0.06)$ \\
\hline$\rightarrow$ & 0.18 & $(0.12-0.24)$ \\
\hline$\longrightarrow$ & 0.08 & $(0.00-0.15)$ \\
\hline$\neg$ & 0.09 & $(0.05-0.13)$ \\
\hline & 0.08 & $(-0.07-0.22)$ \\
\hline & 0.10 & $(0.06-0.13)$ \\
\hline$\rightarrow$ & 0.03 & $(-0.01-0.08)$ \\
\hline & 0.03 & $(-0.01-0.08)$ \\
\hline
\end{tabular}

- Point estimate for mutation incidence associated with each study, and the bars span the $\mathrm{Cl}$.

$\checkmark$ The north-south vertices of the white diamond indicate overall mean point estimate, and eastwest vertices represent the $\mathrm{Cl}$.

Note: Weights are from random-effects analysis.

aThe overall mutation incidence was calculated using the DerSimonian and Laird random-effects model. ${ }^{15}$

$C I=$ confidence interval; $T K I=$ tyrosine kinase inhibitor.

MCyR, and the other patient failed to reach or maintain CCyR. One of the studies identified T315I mutation in 7 patients (incidence $=1.9 \%$ ) who failed treatment due to discontinuation, treatment switch, disease progression, or death. The T315I mutation was reported in $63.6 \%$ (7 of 11) of patients who were exposed to dasatinib and experienced mutations. An F317L mutation was observed in 2 patients $(0.6 \%)$ who experienced disease progression. ${ }^{16}$ The overall mutation incidence was $1.65 \%$ (95\% CI=0.0\%-4.3\%) for dasatinib-treated patients. $\mathrm{I}^{2}$ was high for dasatinib studies at $82.4 \%$.

A single study of nilotinib as first-line treatment included 61 patients, in whom the mutation incidence was 3.3\% (95\% $\mathrm{CI}=0.0 \%-7.7 \%$; Appendix B, available in online article). ${ }^{19}$ The mutations E255K and F359C were reported for this patient cohort, and these patients experienced disease progression.

\section{Imatinib Subgroup Analyses}

The majority of imatinib studies identified failed patients as failing to achieve CHR by 3 months, loss or no cytogenetic response by 6 months, no partial cytogenetic response by 12 months, or a close variation of this definition. The most common definition of failure had an overall imatinib mutation inci- dence of $3.6 \%\left(95 \% \mathrm{CI}=1.9 \%-5.3 \%, \mathrm{I}^{2}=0 \%, P\right.$ value $\left.=0.853\right)$. Stratified analysis of longer versus shorter follow-up durations found similar estimates of mutation incidence of 9.0\% (95\% $\mathrm{CI}=4.4 \%-13.7 \%, \mathrm{I}^{2}=77.1 \%, P$ value $\left.=0.002\right)$ and $9.8 \%(95 \%$ $\mathrm{CI}=1.1 \%-18.5 \%, \mathrm{I}^{2}=83.5 \%, P$ value $=0.002$ ), respectively.

We also generated subgroup analyses of patients by phase, where $\mathrm{CP}$ patients had an overall mutation incidence of $8.7 \%\left(95 \% \mathrm{CI}=4.7 \%-12.7 \%, \mathrm{I}^{2}=71.5 \%, P\right.$ value $\left.=0.004\right)$, while patients in $\mathrm{CP}, \mathrm{AP}$, or BP had an overall mutation incidence of $12.4 \%\left(95 \% \mathrm{CI}=3.6 \%-21.3 \%, \mathrm{I}^{2}=89.4 \%, P\right.$ value $\left.=0.0001\right)$.

\section{Discussion}

When all studies were considered, we found that the overall estimated incidence $(95 \% \mathrm{CI}$ ) of genetic mutation after initial exposure to TKIs in CML was significantly higher among patients exposed to imatinib $(9.7 \%, 95 \% \mathrm{CI}=0.0 \%-13.3 \%)$ compared with dasatanib (1.7\%, 95\% CI=0.0\%-4.3\%), and nilotinib (3.3\%, $95 \% \mathrm{CI}=1.2 \%-7.7 \%, P$ value $<0.001)$. The most commonly identified mutations in imatinib studies were E255K, T315I, M351T, F359V, and G250E. Mutations were identified for T315I, V299L, F317L, and F317I among dasatinib studies, and the mutations E255K and F359C were reported in a single nilotinib study. 
Of the many point mutations identified to date, the mutations highlighted in the National Comprehensive Cancer Network (NCCN) guidelines are E255K/V, V299L, T315A/I/V, F317C/I, and F359C/I/V. ${ }^{11}$ The most commonly identified mutations in our analysis of studies of first-line treatment of CML with TKIs were E255K, T315I, M351T, F359V, and G250E. The mutations E255K, T315I, and F359V were identified as clinically relevant mutations by the NCCN guidelines, which list them in a table with treatment algorithms based on the specific mutation found. The mutation T315I has been reported to range from $4 \%$ to $15 \%$ of patients with imatinib resistance ${ }^{10}$ and, along with other contributing factors, may lead to a poor outcome in these patients. ${ }^{20}$ We estimated this mutation occurred in $13 \%$ of patients who experienced mutations after exposure to imatinib. In addition, we also found M351T and G250E to be among the commonly identified mutations in patients, although these are not listed in the NCCN guidelines. Most patients who had these mutations failed treatment, and they experienced disease progression, which may suggest an association between presence of these mutations and CML progression.

Mutations that are clinically relevant to second-generation inhibitors, such as dasatinib and nilotinib, were F317L/I/C/V, V299L, and T315A for dasatinib; Y253H, E255K/V, and F359V/C for nilotinib; and T315I for both TKIs. ${ }^{21}$ Although rarely found in imatinib-treated patients, current clinical data suggest that V299L is associated with dasatinib resistance. ${ }^{22-24}$ V299L was detected at a incidence of $0.6 \%$ in this metaanalysis in comparison with $1 \%$ in other treatment-resistant patients. ${ }^{25,26}$ One patient with this mutation failed to reach or maintain MCyR, and the other patient failed to reach or maintain CCyR. The T315I and F317L/I mutations were detected in the dasatinib patient cohort, and E255K and F359C were identified in nilotinib-treated patients. Interestingly, most of the patients treated with a second-generation TKI were included in the last category of treatment failure, encompassing treatment discontinuation, treatment switch, disease progression, and/or death. This association suggests that some patients may have a mutation that will confer resistance to the inhibitor even if they are treated with a second-generation TKI.

Progression to AP or BP is most rapid in the first 2 to 3 years of therapy, with decreases in failure rates as treatment continues. ${ }^{27-29}$ Therefore, it is important to detect any mutations that may lead to treatment resistance and disease progression early in treatment. Monitoring of BCR-ABL transcript levels has proven effective in defining patient response and can guide treatment decisions. Point mutations in the BCR-ABL kinase domain can lead to imatinib resistance and are responsible for treatment failure in many cases. ${ }^{30-32}$ The European LeukemiaNet expert panel recommends that mutation analysis should be considered in patients who have suboptimal response or have had a loss of cytogenetic or molecular response at any time. ${ }^{9}$ Other resistance mechanisms have been shown or hypothesized to intervene in resistant patients who do not have BCR-ABL kinase domain mutations. ${ }^{10}$ More than 1 factor may determine the resistant phenotype, and treatment decisions for a second-line strategy should not only include detection of specific mutations but also patient history, risk factors, and comorbidities. ${ }^{11,23}$

\section{Limitations}

A limitation of our review was that, despite imposing inclusion criteria, there were differences in the study designs such as duration of exposure to medication, treatment failure, disease progression, and assay methodology.

Heterogeneity was identified in our meta-analysis, which suggests it may not be appropriate to combine studies. Thus, tables and subgroup analyses that separately show results by each study were also reported. Subgroup analyses showed that 2 sources of heterogeneity in studies were the definition of treatment failure and inclusion of patients in more advanced phases of CML. Although definitions of failure and suboptimal response during imatinib therapy have been published, studies used different definitions, allowing various milestones in therapy. Moreover, patients in AP or BP usually have more mutations than $\mathrm{CP}$ patients, and this was also confirmed by the subgroup analysis.

Within some studies, it was difficult to determine how disease progression was defined and whether the patient was advancing to AP, BP, or death. It is known that mutation frequency varies with disease stage, and including all 3 stages may be a potential confounder in the study. Some studies did not necessarily distinguish between suboptimal response and optimal response or failure. Therefore, the definition of failure may have included suboptimal response for some studies, while others may have excluded it. One study did not test for certain mutations, such as D276G and F359K..$^{33}$ Another study only sequenced the kinase and ATP-loop (amino acid 242 to 395), ${ }^{13}$ while other studies found mutations outside of this amino acid range. Therefore, these studies may underestimate the percentage of these specific mutations.

Although achieving certain milestones at specific time points may predict better response to therapy as recommended by NCCN, this information was not always reported. Failure to achieve cytogenetic disease control in the first 6 months of therapy often reflects the presence of mutations or a high probability that mutations will subsequently be detected. ${ }^{20}$ Thus, imatinib therapy may be most effective when initiated early in the disease course, and molecular and cytogenetic monitoring in the first months of therapy may play a role in identifying patients at high risk of resistance. Molecular and cytogenetic monitoring may identify candidates suitable for more aggressive therapy. In addition, the inherent lack of response by certain BCR-ABL mutations to specific TKIs mutations reflects the need to better understand the TKI regimen selection. 
We had limited information on the achievement of milestones in TKI therapy and the need for therapy change or intensification, and it was difficult to differentiate among patients who were subject to dose escalations for imatinib or the other TKIs. Some patients may have received a higher dose of imatinib, and they may have overcome treatment resistance by dose escalation. We assumed that treatment discontinuation was equated with treatment failure. However, therapy may be discontinued for reasons other than disease progression or treatment resistance, such as discontinuation due to side effects despite attainment of positive endpoints (i.e., CCyR). If so, estimates of treatment failure would be slightly inflated, but they are unlikely to systematically differ by treatment. In addition, some studies had longer follow-up times compared with others with shorter durations. Longer follow-up times may improve the likelihood of detecting mutations. Lastly, the small number of studies and sample sizes limited our ability to identify and estimate genetic mutation rates, particularly for newer TKIs (e.g., the single study of nilotinib as first-line treatment that included only 61 subjects).

\section{Relevance of Mutation Testing}

The current NCCN guidelines recommend performing a mutational analysis after treatment failure, suboptimal response, or disease progression. ${ }^{11}$ Testing patients before or earlier in TKI therapy may lead to earlier detection of mutations and can guide clinicians to other treatments before resistance or before patients progress to more advanced phases. In addition, further research and time are needed to determine whether new therapeutic alternatives to imatinib are effective in controlling drug resistance. ${ }^{34}$

Previous studies have shown the lack of mutation testing in routine clinical practice. ${ }^{35}$ With the high cost of TKIs, it potentially becomes important to perform BCR-ABL kinase domain mutation tests to ensure that patients respond to therapy. The current prices of imatinib and other TKIs have been deemed so high that payers may not be able to afford them. ${ }^{36,37}$ Consequently, it may be relevant to perform a mutation analysis if some patients may have to be exposed to unnecessary costly treatment. Given the numerous considerations involved in selecting a preferred treatment, including the impending generic availability of imatinib, cost-effectiveness analysis of genotype-guided treatment will be complicated but necessary to better inform patient management from a payer perspective.

With the advances in sequencing technology that identify more mutations, the results likely represent more conservative estimates of mutation rates. In addition, the present study examined mutation rates after first-line therapy, and mutation rates are higher in second- and third-line therapy.

\section{Conclusions}

Among patients who received TKIs as first-line therapy for CML, the point estimates for incidence of treatment failure where a BCR-ABL kinase domain mutation was identified ranged from $1.7 \%$ (dasatinib) and $3.3 \%$ (nilotinib) to almost $10 \%$ for imatinib. The most common mutations (> 1\%) were T315I, E255K, and M351T. Because imatinib will soon become generically available, it remains to be seen how prominent a role economics will play in guiding treatment decision making in selection of TKIs in the first- and second-line treatment of CML. Further research is needed to more precisely estimate the incidence of BCR-ABL kinase domain mutations as more clinical studies become available and to understand the costeffectiveness of genotype-guided treatment selection in CML.

\section{Authors}

IULIA D. URSAN, PharmD, is Health Economics and Outcomes Research Fellow; RUIXUAN JIANG, BS, is a Fourth Year Pharmacy Student; TODD A. LEE, PharmD, PhD, is Associate Professor; and A. SIMON PICKARD, PhD, is Associate Professor, Department of Pharmacy Systems, Outcomes, and Policy, College of Pharmacy, University of Illinois at Chicago. DANIEL NG, PharmD, MBA, is Adjunct Assistant Professor, Department of Pharmacy Systems, Outcomes, and Policy, College of Pharmacy, University of Illinois at Chicago, and was Associate Director, Ariad Pharmaceuticals, Cambridge, Massachusetts, at the time of this study. EVAN M. PICKARD, was a High School Senior, Marmion Academy, Aurora, Illinois, at the time of this study.

AUTHOR CORRESPONDENCE: A. Simon Pickard, PhD, Associate Professor, University of Illinois College of Pharmacy, $833 \mathrm{~S}$. Wood St., Rm. 254, M/C 871, Chicago, IL 60612. Tel.: 312.413.3357; Fax: 312.996.0424; Email: pickard1@uic.edu.

\section{DISCLOSURES}

Ursan was supported by a fellowship funded by Takeda Pharmaceuticals Jiang was supported by funding from the Riback Fellowship. A.S. Pickard, Lee, and Ng obtained funding for this study from Ariad Pharmaceuticals. Ng was employed by Ariad at the time of this study.

Study concept and design were contributed by A.S. Pickard, Ng, and Jiang, with assistance from Lee, E. Pickard, and Ursan. Jiang, E. Pickard, Ursan, and A.S. Pickard collected the data, which were interpreted by A.S. Pickard, Lee, and Ursan, assisted by Jiang, E. Pickard, and Ng. The manuscript was written by Ursan and A.S. Pickard, assisted by Lee, Jiang, and E. Pickard. Ursan was primarily responsible for manuscript revision, along with A.S. Pickard, Lee, and Jiang.

\section{REFERENCES}

1. Jabbour E, Kantarjian H. Chronic myeloid leukemia: 2012 update on diagnosis, monitoring, and management. Am J Hematol. 2012;87(11):1037-45.

2. Duffy MJ. The war on cancer: are we winning? Tumour Biol. 2013;34(3): 1275-84.

3. O'Brien SG, Guilhot F, Larson RA, et al. Imatinib compared with interferon and low-dose cytarabine for newly diagnosed chronic-phase chronic myeloid leukemia. N Engl J Med. 2003;348(11):994-1004.

4. Hochhaus A, O'Brien SG, Guilhot F, et al. Six-year follow-up of patients receiving imatinib for the first-line treatment of chronic myeloid leukemia. Leukemia. 2009;23(6):1054-61.

5. Bixby D, Talpaz M. Seeking the causes and solutions to imatinib-resistance in chronic myeloid leukemia. Leukemia. 2011;25:7-22. 


\section{Emergence of BCR-ABL Kinase Domain Mutations Associated with Newly Diagnosed Chronic Myeloid Leukemia: A Meta-Analysis of Clinical Trials of Tyrosine Kinase Inhibitors}

6. Kantarjian HM, Hochhaus A, Saglio G, et al. Nilotinib versus imatinib for the treatment of patients with newly diagnosed chronic phase, Philadelphia chromosome-positive, chronic myeloid leukaemia: 24-month minimum follow-up of the phase 3 randomised ENESTnd trial. Lancet Oncol. 2011;12:841-51

7. O'Hare T, Eide CA, Deininger MW. Bcr-Abl kinase domain mutations, drug resistance, and the road to a cure for chronic myeloid leukemia. Blood. 2007;110:2242-49.

8. Bixby D, Talpaz M. Mechanisms of resistance to tyrosine kinase inhibitors in chronic myeloid leukemia and recent therapeutic strategies to overcome resistance. Hematology Am Soc Hematol Educ Program. 2009;461-76.

9. Soverini S, Hochhaus A, Nicolini FE, et al. BCR-ABL kinase domain mutation analysis in chronic myeloid leukemia patients treated with tyrosine kinase inhibitors: recommendations from an expert panel on behalf of European LeukemiaNet. Blood. 2011;118(5):1208-15.

10. Bhamidipati PK, Kantarjian H, Cortes J, Cornelison AM, Jabbour E Management of imatinib-resistant patients with chronic myeloid leukemia. Ther Adv Hematol. 2013;4(2):103-17.

11. O'Brien S, Berman E, Borghaei H, et al. NCCN clinical practice guidelines in oncology: chronic myelogenous leukemia. J Natl Compr Canc Netw. 2009;7(9):984-1023

12. Jabbour E, Branford S, Saglio G, Jones D, Cortes JE, Kantarjian HM. Practical advice for determining the role of BCR-ABL mutations in guiding tyrosine kinase inhibitor therapy in patients with chronic myeloid leukemia. Cancer. 2011;117(9):1800-11.

13. Roche-Lestienne C, Soenen-Cornu V, Grardel-Duflos N, et al. Several types of mutations of the Abl gene can be found in chronic myeloid leukemia patients resistant to STI571, and they can pre-exist to the onset of treatment. Blood. 2002;100(3):1014-18

14. Kreuzer KA, Le Coutre P, Landt O, et al. Preexistence and evolution of imatinib mesylate-resistant clones in chronic myelogenous leukemia detected by a PNA-based PCR clamping technique. Ann Hematol. 2003;82(5):284-89.

15. DerSimonian R, Laird N. Meta-analysis in clinical trials. Control Clin Trials. 1986;7(3):177-88.

16. Kantarjian HM, Shah NP, Cortes JE, et al. Dasatinib or imatinib in newly diagnosed chronic-phase chronic myeloid leukemia: 2-year follow-up from a randomized phase 3 trial (DASISION). Blood. 2012;119(5):1123-29.

17. Radich JP, Kopecky KJ, Appelbaum FR, et al. A randomized trial of dasatinib $100 \mathrm{mg}$ versus imatinib $400 \mathrm{mg}$ in newly diagnosed chronic-phase chronic myeloid leukemia. Blood. 2012;120(19):3898-905

18. Cortes JE, Jones D, O'Brien S, et al. Results of dasatinib therapy in patients with early chronic-phase chronic myeloid leukemia. J Clin Oncol. 2010;28(3):398-404

19. Cortes JE, Jones D, O'Brien S, et al. Nilotinib as front-line treatment for patients with chronic myeloid leukemia in early chronic phase. J Clin Oncol. 2010;28(3):392-97

20. He H, Shen Y, Zhu Y, Chen S. Prognostic analysis of chronic myeloid leukemia in Chinese population in an imatinib era. Front Med. 2012:6(2):204-11

21. Branford S, Melo JV, Hughes TP. Selecting optimal second-line tyrosine kinase inhibitor therapy for chronic myeloid leukemia patients after imatinib failure: does the BCR-ABL mutation status really matter? Blood. 2009;114(27):5426-35

22. Soverini S, Gnani A, Colarossi S, et al. Philadelphia-positive patients who already harbor imatinib-resistant Bcr-Abl kinase domain mutations have a higher likelihood of developing additional mutations associated with resistance to second- or third-line tyrosine kinase inhibitors. Blood. 2009;114(10):2168-71.

23. Shah NP, Skaggs BJ, Branford S, et al. Sequential ABL kinase inhibitor therapy selects for compound drug-resistant BCR-ABL mutations with altered oncogenic potency. J Clin Invest. 2007;117(9):2562-69.
24. Wu J, Meng F, Kong LY, et al. Association between imatinib-resistant BCR-ABL mutation-negative leukemia and persistent activation of LYN kinase. J Natl Cancer Inst. 2008;100(13):926-39.

25. Burgess MR, Skaggs BJ, Shah NP, Lee FY, Sawyers CL. Comparative analysis of two clinically active BCR-ABL kinase inhibitors reveals the role of conformation-specific binding in resistance. Proc Natl Acad Sci USA. 2005;102(9):3395-400.

26. Bradeen HA, Eide CA, O'Hare T, et al. Comparison of imatinib mesylate, dasatinib (BMS-354825), and nilotinib (AMN107) in an N-ethyl-Nnitrosourea (ENU)-based mutagenesis screen: high efficacy of drug combinations. Blood. 2006;108(7):2332-38.

27. Alvarado Y, Kantarjian H, O'Brien S, et al. Significance of suboptimal response to imatinib, as defined by the European LeukemiaNet, in the longterm outcome of patients with early chronic myeloid leukemia in chronic phase. Cancer. 2009;115(16):3709-18.

28. Baccarani M, Cortes J, Pane F, et al. Chronic myeloid leukemia: an update of concepts and management recommendations of European LeukemiaNet. J Clin Oncol. 2009;27(35):6041-51.

29. Druker BJ, Guilhot F, O'Brien SG, et al. Five-year follow-up of patients receiving imatinib for chronic myeloid leukemia. N Engl J Med. 2006;355(23):2408-17.

30. Branford S, Rudzki Z, Walsh S, et al. Detection of BCR-ABL mutations in patients with CML treated with imatinib is virtually always accompanied by clinical resistance, and mutations in the ATP phosphate-binding loop (P-loop) are associated with a poor prognosis. Blood. 2003;102(1):276-83.

31. Hochhaus A, Kreil S, Corbin AS, et al. Molecular and chromosomal mechanisms of resistance to imatinib (STI571) therapy. Leukemia. 2002;16(11):2190-96

32. Jabbour E, Kantarjian H, Jones D, et al. Frequency and clinical significance of BCR-ABL mutations in patients with chronic myeloid leukemia treated with imatinib mesylate. Leukemia. 2006;20(10):1767-73.

33. Elnahass YH, Mahmoud HK, Ali FT, et al. ABL kinase domain mutations in imatinib-treated Egyptian patients with chronic myeloid leukemia. J Leuk. 2013;1:106

34. Frame D. New strategies in controlling drug resistance. J Manag Care Pharm. 2007;13(8 Suppl A):13-17. Available at: http://www.amcp.org/data/ jmcp/pages\%2013-17.pdf.

35. Kolibaba KS. Molecular monitoring of response in patients with chronic myeloid leukemia. Manag Care. 2013;22(7):40, 50-61

36. Experts in Chronic Myeloid Leukemia. The price of drugs for chronic myeloid leukemia (CML) is a reflection of the unsustainable prices of cancer drugs: from the perspective of a large group of CML experts. Blood. 2013;121(22):4439-42.

37. Curtiss FR. Pharmacy benefit spending on oral chemotherapy drugs. J Manag Care Pharm. 2006;12(7):570-77. Available at: http://amcp.org/data/ jmcp/contemporary_subjects_570_577.pdf.

38. Soverini S, Martinelli G, Rosti G, et al. ABL mutations in late chronic phase chronic myeloid leukemia patients with up-front cytogenetic resistance to imatinib are associated with a greater likelihood of progression to blast crisis and shorter survival: a study by the GIMEMA Working Party on Chronic Myeloid Leukemia. J Clin Oncol. 2005;23(18):4100-09.

39. Branford S, Seymour JF, Grigg A, et al. BCR-ABL messenger RNA levels continue to decline in patients with chronic phase chronic myeloid leukemia treated with imatinib for more than 5 years and approximately half of all first-line treated patients have stable undetectable BCR-ABL using strict sensitivity criteria. Clin Cancer Res. 2007;13(23):7080-85.

40. de Lavallade H, Apperley JF, Khorashad JS, et al. Imatinib for newly diagnosed patients with chronic myeloid leukemia: incidence of sustained responses in an intention-to-treat analysis. J Clin Oncol. 2008;26(20):3358-63. 41. Wei Y, Hardling M, Olsson B, et al. Not all imatinib resistance in CML are BCR-ABL kinase domain mutations. Ann Hematol. 2006;85(12):841-47. 
Emergence of BCR-ABL Kinase Domain Mutations Associated with Newly Diagnosed

Chronic Myeloid Leukemia: A Meta-Analysis of Clinical Trials of Tyrosine Kinase Inhibitors

\section{APPENDIX A Mutation Incidence for Dasatinib}

Criteria Defining Treatment Failure

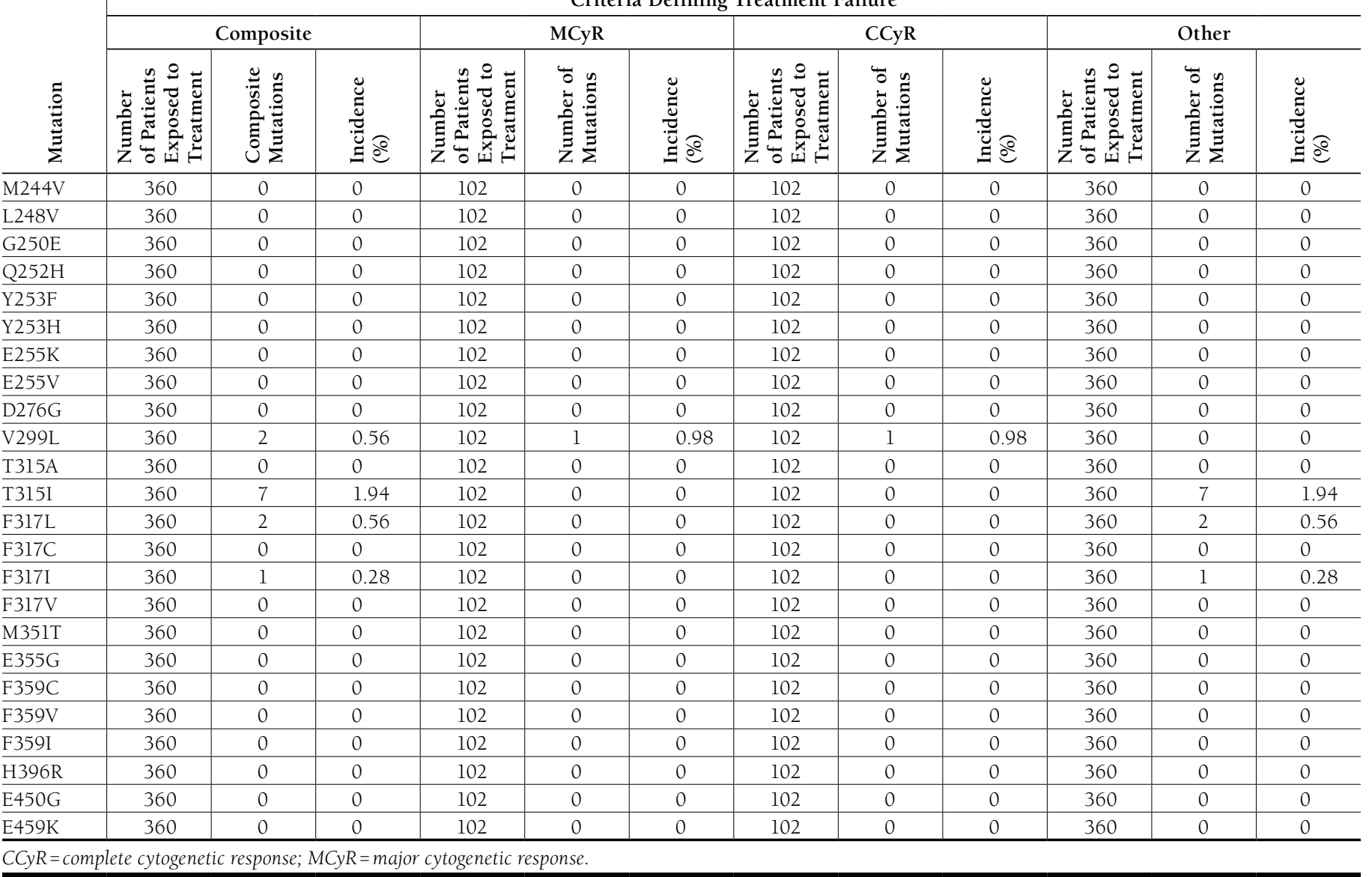


Emergence of BCR-ABL Kinase Domain Mutations Associated with Newly Diagnosed

Chronic Myeloid Leukemia: A Meta-Analysis of Clinical Trials of Tyrosine Kinase Inhibitors

\section{APPENDIX B Mutation Incidence for Nilotinib}

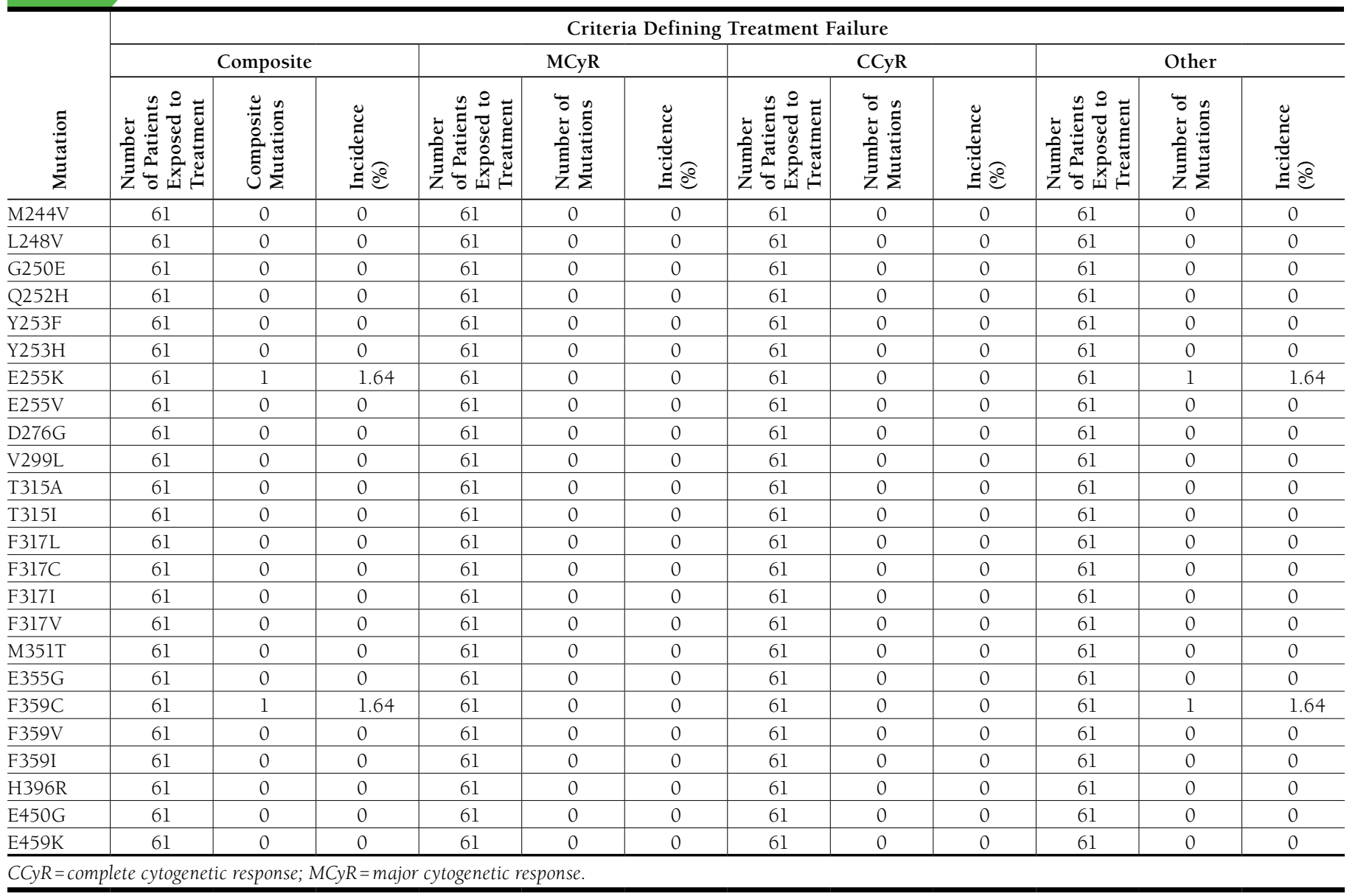

